\title{
Medizinische Betreuung à la française 3
}

\section{Peter Marko}

Dr. med., Mitglied FMH

An Ostern kamen wir dazu, mit der Bahn nach Fréjus zu fahren, um endlich die Ferienwohnung von unserem Sohn Daniel und seiner Frau Barbara in SaintRaphaël an der Côte d'Azur zu bewundern. Sowohl gemäss den Angaben im Internet wie auch des Bahnanzeigers sollte Fréjus die Endstation sein. Arglos stiegen wir abends in der Dunkelheit in Nizza in den Zug. Hinter uns sass ein Mann mit einem Mädchen, der hielt sich die linke Hand und jammerte dauernd. Nach einer Weile fragte ihn meine Frau Silvia, was mit seiner Hand sei. Er zeigte sie und erklärte, er habe einen Gichtanfall. In meinen langen Praxisjahren sah ich nur in Atlanten ein solches Bild. Nicht nur die Finger waren von Harnsäureablagerungen verformt und gerötet. Wir empfahlen ihm, gleich zum Arzt zu gehen, da er keine richtigen Medikamente gegen einen Gichtanfall hatte. Bald darnach stieg er sowieso aus. Wir ahnten nicht, dass diese Begebenheit ein schlechtes Zeichen war.

\section{Raus- und aufgeschmissen}

Als wir in Fréjus eintrafen und niemand Anstalten machte, auszusteigen, kam uns dies merkwürdig vor. Wir sahen kein Bahngebäude und fragten die Mitfahrer, ob wir in Fréjus seien, was sie bejahten. Warum

\section{Die Türen schlossen sich und spuckten meine Frau Silvia aus mehr als einem Meter Höhe auf den asphaltierten Weg.}

steigt denn niemand aus? Sie würden halt weiterfahren, antworteten sie. Kann man das denn? Ja, lautete die Antwort. Silvia ging zur Türöffnung, um auf den Bahnsteig zu schauen, während ich mit dem grossen Koffer unruhig sitzen blieb. Als sie zwischen den Türflügeln stand, wurden ihre Unentschlossenheit und ihr Zögern energisch beendet - die Türen schlossen sich und spuckten sie aus mehr als einem Meter Höhe auf den asphaltierten Weg. Ich blieb ohne Geld, Handy und Ahnung, in welchem Hotel wir übernachten sollten, und mit meinen rudimentären Französischkenntnissen im Zug. Meine Unruhe stieg. Silvia wurde raus-, ich fühlte mich aufgeschmissen. Zum Glück bemerkten nicht nur die Ausgestiegenen, sondern auch der Lokführer, was passiert war, weshalb er nicht losfuhr. Die Türe wurde wieder geöffnet, so dass ich aussteigen und mich um meine Frau kümmern konnte, die inzwischen aufgestanden war und sich frei bewegte. An-

Ich benutzte ihren Schal, den sie zufällig bei sich trug, um das Handgelenk notdürftig zu fixieren.

stelle des Mannes mit dem Gichtanfall, hielt sie nun ihr linkes Handgelenk. Sie klagte über Schmerzen und lachte, jetzt sei sie daran, den Arm zu halten und zu jammern. Ich benutzte ihren Schal, den sie zufällig bei sich trug, um das Handgelenk notdürftig zu fixieren. Sofort tat es ihr auch weniger weh. Wir gingen zu Fuss ins nahegelegene Hotel. Unterwegs realisierten wir, dass gegenüber der Stelle, an der wir ausgestiegen waren, etwas erhöht auf der anderen Seite der Geleise, sich ein hübscher Jugendstilbahnhof befand. Aus dem Zug hatten wir ihn nicht sehen können.

Im besseren Licht unseres Hotelzimmers schaute ich die Hand an. Im Bereich des Kahnbeins fand sich eine blaue, weiche Schwellung, die sehr druckempfindlich war. Auch die Bewegungen im Handgelenk schmerzten dort. Da wir keine Binden bei uns hatten, fixierten wir das Gelenk wieder mit dem Schal und beschlossen, Barbara und Daniel nicht mit einem Anruf unnötig zu beunruhigen. Daniel sollte uns ohnehin am nächsten Vormittag um halb zehn im Hotel abholen. Er könnte uns dann gleich mit dem Auto ins nächste Spital bringen.

\section{Auf ins nächste Spital}

Die Nacht war nicht schlecht, aber die Schmerzen in der Hand waren am nächsten Morgen unverändert. Auf der Notfallstation waren schon zwei Patienten. Gleich nach der Aufnahme der Personalien wurde Silvia aufgefordert, einer Pflegefachfrau zu folgen. Als wir sie begleiten wollten, sagte die Frau: nein, nur die Patientin dürfe mit ihr rein. Wir blieben im schönen, warmen Wetter draussen auf dem Parkplatz vor der Not- 
fallstation. Nachdem wir die wichtigsten Neuigkeiten und Probleme besprochen hatten, kam uns nach anderthalb Stunden wieder in den Sinn, warum wir eigentlich hergekommen waren. Wir gingen zum Empfang, aber niemand war dort, und wir fanden auch

\section{Dass die Behandlung so lange dauerte, will Silvia mir auch heute noch nicht glauben.}

keine Glocke, mit der wir uns hätten bemerkbar machen können. Wir schöpften Hoffnung, als die Ambulanz mit einem liegenden, aber offensichtlich nicht schwer kranken Menschen kam. Leider gelang es uns nicht, mit ihnen ins Gespräch über unsere Patientin zu kommen. Wir waren verzweifelt. Nach einer weiteren Stunde, also nach insgesamt mehr als zweieinhalb Stunden, erschien Silvia in einer merkwürdigen Montur, ihre Bluse und Jacke in der rechten Hand haltend, gut gelaunt. Auffallend war ein klobiger Gips bis hinter den Ellbogen. Sie war nicht bereit, in dieser Aufmachung in die Stadt zu fahren, auch wenn das "Gwändli" sicher von einem Modehaus in Paris entworfen worden war und ihr nicht schlecht stand. Wir kehrten ins Hotel zurück, wo sie sich ein Reservehemd von mir anzog, denn der Gips war für ihre Garderobe zu dick.

Dass die Behandlung so lange dauerte, will Silvia mir auch heute noch nicht glauben. Jedenfalls berichtet sie, dass sie zunächst in einem Gang sitzen gelassen wurde. Das Treiben dort sei interessant gewesen, aber als sie des Wartens dann doch überdrüssig geworden sei, habe sie sich bemerkbar gemacht. Danach sei sie zuerst ins Röntgen, dann in einen Raum geführt worden, wo drei Personen mühsam und lange versucht hätten, ihr

Bitte entschuldigen Sie die Qualität des Fotos. Leider ist das Original nicht mehr verfügbar, da das Smartphone mit den entsprechenden Daten vor kurzem in Frankreich verlorenging. Wir (der Autor und die Redaktion) wollten dennoch nicht auf die Abbildung verzichten

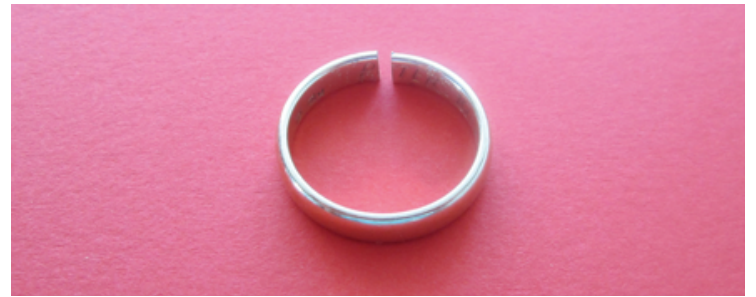

Schade: Silvias Ehering hat die Prozedur nicht unbeschadet überstanden.

den Ehering abzunehmen. Sie hatten offenbar nicht das geeignete Instrument dazu, wie wir es für solche Fälle in unserer Praxis verwenden. Und die Eheringe in Frankreich sind wahrscheinlich dünner, auch wenn

\section{Das Gipsen ging gemäss Silvias Bericht zügig voran.}

unsere nicht besonders dick sind und genau der Schweizer Norm entsprechen (s. Bild). Das Gipsen ging gemäss ihrem Bericht zügig voran, und das «Gwändli» mussten sie auch nicht lange suchen und anpassen. Es ist einheitlich, eben égalité. Eigentlich war Silvia mit dem Ganzen zufriedener als der Rest der Familie, was auch ihr Lächeln auf dem Foto bezeugt.

\section{Nach der Entlassung}

Auf dem Röntgenbild sah man keinen Knochenbruch, was bei einem frischen Kahnbeinbruch nicht selten ist. Auch das Computertomogramm zeigte bei der Kontrolle nach einer Woche zu Hause keinen solchen Bruch, aber auf dem üblichen Röntgenbild einen ins Gelenk reichenden, nicht verschobenen Speichenbruch, der problemlos nach sechs Woche verheilte.

Die Rechnung für die ganze Behandlung, also die Röntgenaufnahmen, das Gipsen, ein kleines Zeugnis, das "Gwändli» nicht zu vergessen, betrug 74 Euro. Für unsere Krankenkasse gilt: Im Falle eines Falles in Frankreich fallen.

Daniel möchte, dass wir ihn und seine Frau wieder in Frankreich besuchen. Sollen wir es wagen?

\section{Bildnachweis}

Porträtfoto von Silvia Marko: Daniel Rüfenacht Foto vom Ehering: Silvia Marko

\section{Quelles expériences avez-vous faites?}

Vous est-il vous aussi arrivé, en tant que médecin, de vous retrouver tout à coup dans la peau d'un patient? Si votre histoire vous semble susceptible d'intéresser les lecteurs du BMS, n'hésitez pas à envoyer un article de 8000 signes maximum à l'adresse redaktion.saez[at]emh.ch - en cas d'évaluation positive par la rédaction, votre manuscrit sera publié dans le cadre de la série "Quand le médecin devient patient». 